\title{
The LMC Intermediate and Old Populations
}

\author{
Edward W. Olszewski \\ Steward Observatory, University of Arizona, 933 N. Cherry, Tucson, \\ $A Z$ 85721, USA
}

\begin{abstract}
This review concentrates on the oldest populations in the LMC. Specifically, the LMC has a large extent and mass, it has star clusters coeval with those in the Milky Way halo, and more work needs to be done before we are convinced that there are similarly-aged field stars in the LMC. Further, all studied fields in the Clouds are different from Milky Way halo fields. Observations with the new generation of large telescopes can clear up the uncertainties noted in this review.
\end{abstract}

\section{Preamble}

Before we can discuss the halo population of the LMC we have to agree on what we mean by that term. It is not semantics, because the connotations of the term "halo" rest upon how we think the Milky Way evolved. Those connotations may not apply to other galaxies. Does the LMC have any clusters or field stars that are demonstrably the same age and metallicity as a typical metal-poor "old halo" globular cluster in the Milky Way? Since LMC field stars have now been traced to large distances from the LMC bar, does "halo" mean "far from the center," or "in an extended z-direction distribution" and/or "supported by its own velocity dispersion?" Even with such limited definitions it is difficult to place the oldest stars in the LMC comfortably into a simple definition of halo. This discussion has particular relevance in 1998 because the interpretation of the microlensing results (there was heated discussion at the Third Stromlo Symposium in August 1998) depends on the structures of the Magellanic Clouds themselves. We need to know if the LMC has no halo or a spherical halo, or if the LMC has significant outer structure or warps. Guides to the earlier literature can be found in Olszewski et al. (1996) or in Westerlund (1997).

\section{Mass and Extent of LMC}

What do we know about the mass and extent of the LMC? While we tend to think of the LMC as only a few kpc in size (the "SN 1987A" view), a very large size, perhaps $14^{\circ} \times 16^{\circ}\left(1^{\circ}\right.$ is about $\left.0.9 \mathrm{kpc}\right)$, of the LMC has been known for a long time (summarized in de Vaucouleurs \& Freeman 1973). In more recent times, Bothun \& Thompson (1988) used a "parking lot" CCD camera to measure $\mathrm{D}_{25}=9.1^{\circ}$, a disk scale length of $1.67^{\circ}$, and a diameter of at least $10^{\circ}$. Irwin (1991) estimated a size of $23^{\circ} \times 17^{\circ}$ from star counts, and showed that the 
"halos" of the two Clouds essentially overlap. Nikolaev \& Weinberg (1998) have estimated the LMC tidal radius $(\sim 11 \mathrm{kpc})$ based on USNO-A starcounts and on prototype 2MASS star counts. They then derive an LMC mass of $>1 \times 10^{10}$ $\mathrm{M}_{\odot}$, given that the Milky Way's mass is $>10^{12} \mathrm{M}_{\odot}$.

Suntzeff et al. (1992) showed that the Reticulum (11.4 $4^{\circ}$ from LMC bar, or $10 \mathrm{kpc})$ and NGC $1841\left(14.9^{\circ}, 13 \mathrm{kpc}\right)$ clusters had velocities consistent with LMC membership. Schommer et al. (1992) showed a sparsely populated rotation curve out to the distance of Reticulum. If one assumes that the rotation curve is flat, or if one uses the velocity dispersion of the oldest clusters, one derives a "massive" LMC of $1-2 \times 10^{10} \mathrm{M}_{\odot}$. Such a galaxy has a luminous halo or dark halo or both. Kunkel et al. (1997) have measured the velocities of hundreds of newly discovered $\mathrm{C}$ stars out to beyond $10 \mathrm{kpc}$ and have estimated an LMC mass of $0.6-1.5 \times 10^{10} \mathrm{M}_{\odot}$. The velocity dispersion, $\sim 30 \mathrm{~km} \mathrm{~s}^{-1}$, of the oldest star clusters does imply a distribution with a $\mathrm{z}$ scale height of of a few kpc. It is known, for example in Walker (1991), that some clusters are at different distances from the fields on which they are projected, or are at different distances from the best-fit planar model. It was also noted by Schommer et al. (1992) that the velocities of some of the oldest clusters projected on the bar of the LMC cannot fit the planar model. The velocity dispersion of the Kunkel et al. C stars, $15 \mathrm{~km} \mathrm{~s}^{-1}$, however, is not what is expected for a spherical LMC halo. These stars are presumably drawn from an intermediate-age population.

Other tracers of the $\mathrm{z}$ extent of the LMC halo may be old long-period variables with dispersion $33 \mathrm{~km} \mathrm{~s}^{-1}$ (Hughes et al. 1991, but see discussion in Olszewski et al. 1996), or the old, rather uncertain result (Freeman, unpublished) of an RR Lyrae velocity dispersion of $\sim 50 \mathrm{~km} \mathrm{~s}^{-1}$. One can also use the dispersion of the magnitudes of RR Lyrae stars in the older photographic surveys (Graham 1973, see also Walker 1991), or the dispersion in magnitudes of the clump giants. A change in stellar populations may make an important change in the measured magnitude dispersions, although this effect is not well quantified. The more modern all-LMC surveys, if well calibrated, could place useful limits on the depth of the LMC. We await those results eagerly since the older work is based on too few stars to place meaningful limits on the depth of the LMC.

Finding stars distant from the LMC center does not guarantee that we are seeing a halo population. Freeman et al. (1983) first noted that all populations, including that of the oldest clusters, were in a rotating system. Schommer et al. (1992) measured high-quality velocities for these and other objects, and showed that all objects, regardless of age, have the same disk rotation curve. This result is very different from that of the Milky Way or of M33, for example (Schommer et al. 1991). Is this set of strange kinematics the result of late formation of "halo objects," or is there another explanation (Weinberg 1999)?

\section{Ages of the Oldest Clusters}

\subsection{Direct Age Determinations}

Olsen et al. $(1998,1999)$ and Johnson et al. (1999) have used WFPC2 images to derive the ages of eight of the oldest clusters in the LMC. They age-dated the clusters by comparing the CMDs to those of galactic globulars of the same 
metallicity. No differences were found, leading both groups to deduce that the oldest LMC clusters are the same age as the oldest Milky Way clusters to $\pm 1 \mathrm{Gyr}$. Although there have been papers claiming, based on color-magnitude diagrams, that some of these LMC clusters were indeed the same age (or younger, for that matter) as Milky Way clusters, the papers by Olsen et al. and Johnson et al. are the first that have the photometric accuracy to make that claim at the \pm 1-2 Gyr level. These two papers show that 8 old LMC clusters observed to date with HST, both in the LMC bar and in the outer regions of the LMC, are indistinguishable in age from the older Milky Way clusters.

Many authors, myself included, had expected that these clusters would be 2-3 Gyr younger than Milky Way globulars (see Da Costa 1993 and Olszewski et al. 1996 as examples). Da Costa (1999) argues that some of these LMC clusters have horizontal branch morphologies consistent with their derived ages, while the radially more distant LMC clusters seem to show the same sort of second-parameter effects found in outer halo clusters in the Milky Way. He also argues that some of these 8 clusters measured with HST have very similar HB morphologies but abundances that vary by $0.7 \mathrm{dex}$, and therefore wonders if metallicity is even the first parameter in the LMC.

\subsection{Is the Set of Oldest Clusters Complete?}

There are several reasons to believe that the list of 13 old clusters found in Table 6 of Suntzeff et al. (1992) is complete. First, estimates of LMC cluster destruction timescales (see discussion in Olszewski 1993) show that, unlike the case for the Milky Way, even modest LMC clusters can survive a Hubble time. Second, Mateo (1993) has shown that fading models predict that the brighter of the current blue, young clusters will still be luminous enough to be in cluster catalogs at an age of $15 \mathrm{Gyr}$. Third, there is a demonstrable cluster age gap (see Olszewski et al. 1996), even if the edges of that gap are uncertain at the $\sim 1$ Gyr level (Sarajedini 1998). Fourth, Olszewski and collaborators (Olszewski 1993) showed that, for random clusters without age determinations, there are fewer clusters than expected at the metallicity of clusters that would be in the age gap. And fifth, Da Costa (1991) and Geisler et al. (1997) found no new age-gap clusters in their searches. The Geisler et al. study targeted clusters thought to be in the age gap from integrated photometry and from Olszewski and collaborators' metal abundances. We can therefore conclude that the Olsen et al. and Johnson et al. results are representative of the LMC old cluster system as a whole. The cluster age gap remains important for comparing LMC star formation histories as derived from clusters and from field stars.

\section{Ages of the RR Lyraes}

RR Lyrae stars are old, but are they ancient? If we see an RR Lyrae star in the LMC, can we conclude that it is " 15 Gyr old", and can we conclude that it is the same age as an RR Lyrae star in one of the ancient clusters? Could we conclude that there is a group of RR Lyraes in the LMC field that is demonstrably younger than the cluster RR Lyraes, thus showing that deriving the LMC star formation history from clusters is incorrect? 
NGC 121 in the SMC is demonstrably not of "globular cluster age" (Stryker et al. 1985) yet it contains RR Lyraes. There are metal-rich, thick-disk-kinematics RR Lyraes in the Milky Way (Taam et al. 1976) that might be thought to be younger than halo RR Lyraes. There are RR Lyraes in the "young halo" Milky Way globulars (see Da Costa \& Armandroff 1995 for a good list of "young" halo clusters, and see the "Catalog of Variable Stars in Globular Clusters" for the list of variables). Alcock et al. (1996) claim a dearth of high amplitude RR Lyraes in the LMC field, and that what RR Lyraes there are must have formed from a population with a red horizontal branch of fairly low abundance. We must therefore conclude that the presence of an RR Lyrae indicates that the system is "old," as opposed to "ancient." Without additional information, such as the line of sight velocity dispersion of RR Lyraes as a function of abundance, or the relative fraction of blue horizontal branch stars in the LMC field, or some light-curve age diagnostic, we cannot tell if the RR Lyraes are part of the very oldest population in the LMC.

\section{Ages of the Oldest Stars}

Are there any field stars that are demonstrably ancient? One way to attack this problem is to study the color distribution of the clump giants and horizontal branch stars. From the color-magnitude diagrams of LMC and SMC clusters, we can see how the red clump changes into the red horizontal branch as a cluster ages (see Udalski 1998). Gardiner and Hatzidimitriou (1992) used an older version of this relation to derive the fraction of ancient horizontal branch stars in the SMC, concluding that $7 \%$ of the SMC was ancient (this percentage drops substantially if the modern calibration is used, see discussion in Olszewski et al. 1996). Smecker-Hane et al. (1994) showed the existence of a red clump and $\mathrm{a} \mathrm{HB}$ in the Carina $\mathrm{dSph}$, differing [mostly] in magnitude, and coming from populations of differing ages. With good-enough photometry and simple-enough populations, one should be able to quantify the age distribution of the old LMC as seen by the red clump and red horizontal branch.

Finding the LMC blue horizontal branch is more problematic. The BHB is easily hidden by the main sequence of a younger population. There are no LMC fields with a possible BHB that do not also have a younger population. One must therefore rely on the part of the BHB that is distinct from the main sequence, thus requiring better photometry than is available with the ongoing LMC surveys.

We therefore conclude that we have yet to see the population II horizontal branch in the LMC, even if it exists. Searching for old subgiants in the LMC field, with or without the use of HST (Walker 1999 is one example), may be the best way to prove definitively whether the oldest LMC field star population is "old" or "ancient."

\section{Cautionary Tale: The LMC as a not-so-simple System}

Since the dawn of the CCD age the vast majority of the deep photometry of the Clouds has been in the LMC. Probably the major reason is that the LMC was considered simple, while the SMC was considered complicated and crowded. 
However, it now looks as though neither galaxy is well behaved, at least in terms of a halo population, although the SMC might be the simpler galaxy after all. NGC 2257 in the LMC is $~ 8$ degress $\mathrm{N}$ of the bar. The surface brightness in the field surrounding this cluster is $\sim 27 \mathrm{mag} \mathrm{arcsec}^{-2}$. We (see Olszewski 1993) have measured metal abundances of 36 stars in this field. Twenty-one are more metal rich than $[\mathrm{Fe} / \mathrm{H}]=-1.0$. Of the fifteen more metal poor than $[\mathrm{Fe} / \mathrm{H}]=-1.0$, only seven are more metal poor than $[\mathrm{Fe} / \mathrm{H}]=-1.5$. The startling aspect of this field is the high abundance of most of the stars. This must be among the simplest fields in the LMC, yet it is very different from what one would have predicted for a pure halo field. We conclude that the distribution of metallicity in "HST" fields needs to be derived from ground-based spectroscopy of giants in those fields (actually, in larger fields surrounding the tiny HST fields) before one can derive the star formation history. The metallicity distribution of the SMC field NGC 121 (Suntzeff et al. 1986, 1999) seems to me to be equally non-halo-like. It has proven difficult in practice to find fields in the Clouds that are anything like the halo of the Milky Way. It will be fascinating to see which connotations of the word "halo" must be abandoned to find the oldest, least-confined-to-a-plane populations in each Cloud.

\section{Concluding remarks}

The review of Olszewski et al. (1996) concluded with a substantial list of questions that need to be answered before we have a good understanding of the older populations in the Clouds. Many of these problems can now or soon be addressed with the new surveys (MACHO, EROS, Great Circle Camera, OGLE), the new telescopes (Gemini, VLT, Magellan, etc.), and the new multi-object spectrographs (Hydra south, 2dF, etc.), and with HST.

Acknowledgments. EO was partially supported by the NSF. Since this symposium honored Sidney van den Bergh, and the DAO by association, let me thank him and them for the great environment in which to be a postdoc, and for being examples of good scientists and people.

\section{References}

Alcock, C., et al. 1996, AJ, 111, 1146

Bothun, G.D., \& Thompson, I.B. 1988, AJ, 96, 877

Da Costa, G.S. 1991, in "The Magellanic Clouds," IAU Symp. 148, edited by R. Haynes \& D. Milne, p. 183

Da Costa, G.S. 1993, in "The Globular Cluster-Galaxy Connection," edited by G.H. Smith \& J.P. Brodie, ASP Conf Series 48, p.363

Da Costa, G.S., \& Armandroff, T.E. 1995, AJ, 109, 2533

Da Costa, G.S. 1999, this volume

De Vaucouleurs, G., \& Freeman, K.C. 1973, Vistas Astron., 14, 163

Freeman, K.C., Illingworth, G., \& Oemler, A. 1983, ApJ, 272, 488

Gardiner, L.T., \& Hatzidimitriou, D. 1992, MNRAS, 257, 195 
Geisler, D., Bica, E., Dottori, H., Claria, J.J., Piatti, A.E., \& Santor, J.F.C., Jr. 1997, AJ, 114, 1920

Graham, J.A. 1973, in "Variable Stars in Globular Clusters and Related Systems," edited by J.D. Fernie, p.120

Hughes, S.M.G., Wood, P.R., \& Reid, N. 1991, AJ, 101, 1304

Irwin, M.J. 1991, in "The Magellanic Clouds," IAU Symp. 148, edited by R. Haynes \& D. Milne, p. 453

Johnson, J.A., Bolte, M., Bond, H.E., Hesser, J.E., de Oliveira, C.M., Richer, H.B., Stetson, P.B., \& VandenBerg, D.A. 1999, this volume

Kunkel. W.E., Demers, S., \& Irwin, M.J. 1997, ApJ, 488, L129

Mateo, M. 1993, in "The Globular Cluster-Galaxy Connection," edited by G.H. Smit \& J.P. Brodie, ASP Conf Series 48, p. 387

Nikolaev, S., Weinberg, M.D. 1998, BAAS, 192, \#55.03

Olsen, K.A.G., Hodge, P.W., Mateo, M., Olszewski, E.W., Schommer, R.A., Suntzeff, N.B., \& Walker, A.R. 1998, MNRAS, 300, 665

Olsen, K.A.G., Hodge, P.W., Mateo, M., Olszewski, E.W., Schommer, R.A., Suntzeff, N.B., \& Walker, A.R. 1999, this volume

Olszewski, E.W. 1993, in "The Globular Cluster-Galaxy Connection," edited by G.H. Smith \& J.P. Brodie, ASP Conf Series 48, p.351

Olszewski, E.W., Suntzeff, N.B., \& Mateo, M. 1996, ARA\&A, 34, 511

Sarajedini, A. 1998, AJ, 116, 738

Schommer, R.A., Christian, C.A., Caldwell, N., Bothun, G.D., \& Huchra, J. 1991, AJ, 101, 873

Schommer, R.A., Olszewski, E.W., Suntzeff, N.B., \& Harris, H.C. 1992, AJ, 103, 447

Smecker-Hame, T.A., Stetson, P.B., Hesser, J.E., \& Lehnert, M.D. 1994, AJ, 108,507

Stryker, L.L., Da Costa, G.S., \& Mould, J.R. 1985, ApJ, 298, 544

Suntzeff, N.B., Friel, E., Klemola, A.A., Kraft, R.P., \& Graham, J.A. 1986, AJ, 91275

Suntzeff, N.B., Schommer, R.A., Olszewski, E.W., \& Walker, A.R. 1992, AJ, 104,1743

Suntzeff, N.B., Walker, A.R., Smith, V.V., Kraft, R.P., \& Klemola, A. 1999, this volume

Taam, R.E., Kraft, R.P., \& Suntaeff, N.B. 1976, ApJ, 207, 201

Udalski, A. 1998, AcA, 48, 383

Walker, A.R. 1991, in "The Magellanic Clouds," IAU Symp. 148, edited by R. Haynes \& D. Milne, p.307

Walker, A.R. 1999, this volume

Weinberg, M.D. 1999, in proceedings of Third Stromlo Symposium

Westerlund, B.E. 1997, "The Magellanic Clouds," Cambridge Astrophysics Series \#29 


\section{Discussion}

Jim Hesser: Our poster makes some points relevant to the robustness of conclusions about the relative ages of the oldest clusters in the Magellanic Clouds and the Galaxy. We report differential ages for four clusters in the outer halo of the Milky Way at roughly twice the distance of the Clouds. For NGC2419, an [Fe/H] $=-2.2$ cluster, we see no evidence for an age difference relative to the nearby cluster M92. As Harris et al. (1997) pointed out, this suggests that a burst of star and/or star cluster formation occurred over a protogalactic volume of some 200 Kpc diameter. For three intermediate metallicity outer halo clusters with stubby red horizontal branches, so-called second parameter clusters, we find evidence that they are somewhat younger than nearby clusters of comparable metallicity in the sense of Lee, Demarque and Zinn. However these conclusions do depend fairly sensitively on knowing what the relative abundance ratios $([\mathrm{Fe} / \mathrm{H}],[\alpha / \mathrm{Fe}]$, etc.) are in the distant and nearby clusters where we have information of very different quality: high-dispersion spectroscopy for many stars in the nearer objects; and, for more distant clusters, low-dispersion spectroscopy - often just of the $\alpha$-element $\mathrm{Ca}$ - for a few of their most luminous giants, integrated-light photometry or CMD comparisons. To know how the oldest Cloud clusters really compare to Galactic globulars in age we are going to have to get more reliable spectroscopic information for them. 